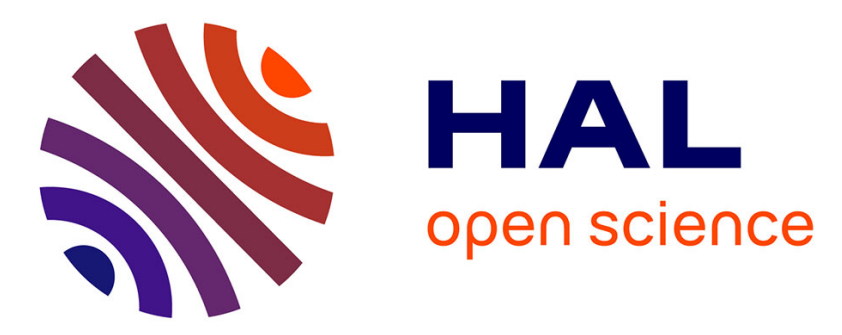

\title{
Community of Practice Theory and Process Modelling: Two Tools for Better Collaboration in Research Projects
}

Virginie Goepp, Charlotte Munzer, Françoise Feugeas

\section{To cite this version:}

Virginie Goepp, Charlotte Munzer, Françoise Feugeas. Community of Practice Theory and Process Modelling: Two Tools for Better Collaboration in Research Projects. IFIP International Conference on Advances in Production Management Systems (APMS), Sep 2014, Ajaccio, France. pp.3-10, 10.1007/978-3-662-44733-8_1. hal-01387101

\section{HAL Id: hal-01387101 \\ https://hal.inria.fr/hal-01387101}

Submitted on 25 Oct 2016

HAL is a multi-disciplinary open access archive for the deposit and dissemination of scientific research documents, whether they are published or not. The documents may come from teaching and research institutions in France or abroad, or from public or private research centers.
L'archive ouverte pluridisciplinaire HAL, est destinée au dépôt et à la diffusion de documents scientifiques de niveau recherche, publiés ou non, émanant des établissements d'enseignement et de recherche français ou étrangers, des laboratoires publics ou privés.

\section{(c)(1)}

Distributed under a Creative Commons Attribution| 4.0 International License 


\title{
Community of practice theory and process modelling: two tools for better collaboration in research projects
}

\author{
Virginie GOEPP ${ }^{1}$, Charlotte MUNZER ${ }^{1}$ and Françoise FEUGEAS ${ }^{1}$ \\ ${ }^{1}$ ICube, INSA de Strasbourg 24, bld de la Victoire 67084 - Strasbourg Cedex France \\ \{virginie.goepp, charlotte.munzer, francoise.feugeas\}@insa- \\ strasbourg.fr
}

\begin{abstract}
Today, research projects are often multi-disciplinary involving several research teams. For such projects to be a success implies, for these teams, to work together in an efficient manner. To improve collaboration we propose to work on two complementary aspects. The first aspect exploits the community of practice theory in order to define the knowledge to share and the way to share it. The second aspect applies process modelling in order to model research processes at different level of granularity (project, task, protocol). In this way, process uncertainty is reduced and a shared vision of the process is worked out. We illustrate our proposition on the SEPOLBE project that involves four research teams and a company to develop bio admixtures for concrete.
\end{abstract}

Keywords: community of practice, process modelling, BPMN, experimental protocol

\section{Introduction}

In today context of fierce competition, research projects are more and more multidisciplinary. This requires the collaboration between several research teams who have different backgrounds and are disseminated in different locations.

The collaboration enables, among other, to share expensive experimental means. However, setting up the collaboration in such a context requires overcoming the barriers linked to location and discipline. For the collaboration to be efficient the project members have to explicitly define the way they have to work together and to share knowledge. In this boarder, the Community of Practice theory and process modelling can be useful tools. The first enables the definition of the kind of knowledge shared and the way to share it. The second enables the formalization of experimental protocols in order to reduce their uncertainty and to provide a shared and unified view of them.

The remainder of the paper is structured as follows. Section 2 deals with Community of Practice theory that we apply to research projects. Section 3 deals with process modelling and BPMN (Business Process Model Notation). Section 4 applies Community of Practice Theory and process modelling to the SEPOLBE research project dedicated to develop bioadmixtures for concrete.

adfa, p. 1, 2011.

(C) Springer-Verlag Berlin Heidelberg 2011 


\section{Community of practice theory}

\subsection{Definition}

The notion of Community of Practice (CoP) has largely been discussed in the literature. For example, Cox in [1] makes a comparative review of four seminal works on CoPs. He shows that the ambiguities of the terms "communities" and "practice" allow the concept to be re-appropriated for different purposes.

In [2] CoPs are treated as the informal relations and understandings developed in mutual engagement on an appropriated joint enterprise. In other words, a community of practice is defined as a group that coheres through "mutual engagement" on an "appropriated enterprise", and creates a common "repertoire". It goes beyond the simple notion of team that not necessarily creates a common "repertoire". In our view, the collaboration between research teams becomes efficient when this team becomes a community of practice and shares knowledge.

In [3] the concept is redefined towards a more managerial stance, making the concept more popular and simple. Here CoPs are "groups of people who share a concern, a set of problems, or a passion about a topic, and who deepen their knowledge and expertise in this area by interacting on an ongoing basis." According to [1], this definition is much vaguer than the previous one. The definition is of a group that is somehow interested in the same thing, not closely tied together in accomplishing a common enterprise. The purpose is specifically to learn and share knowledge, not to get the job done. From this point of view a CoP has three structural features:

1. Domain: it “... creates common ground and a sense of common identity. A welldefined domain legitimises the community by affirming its purpose and value to members and other stakeholders. The domain inspires members to contribute and participate, guides their learning, and gives meaning to their actions" [3].

2. Community: it "...creates the social fabric of learning. A strong community fosters interactions and relationships based on mutual respect and trust. It encourages a willingness to share ideas, expose one's ignorance, ask difficult questions, and listen carefully. Community is an important element because learning is a matter of belonging as well as an intellectual process. [3].

3. Practice: it "...is the specific knowledge the community develops, shares and maintains" [3].

The definition of a CoP is interesting as it enables to consider research projects from a different point of view. In this view, the research project becomes a place in which knowledge is shared and exchanged between the different members involved.

\subsection{Community of practice typologies}

The structural features of a CoP can be refined through corresponding typologies.

Thus, for the domain, McDermott [4] indicates four types of community: (i) communities which are linked to a strategic objective; (ii) communities which focus on tactical processes, process optimisation and sharing of best practices; (iii) projectbased communities and (iv) communities developing a particular body of knowledge. 
A research project is generally a project based community. It is the project in itself that forms the domain of the CoP.

Concerning the community, the CoP theory provides a set of typical roles. Wenger, McDermott and Snyder [3] describe the specific role of "coordinator" as the one who organises events, connects communities and generally keeps the community alive. They identify three levels of participation in a CoP:

- The core group: a small group of people (10 to 15 percent of the $\mathrm{CoP}$ ) that carries out most of the work in the community. It actively participates in discussions and identifies the topics to be addressed within the community. As the CoP matures, this group becomes the coordinator's auxiliary.

- The active group, 15 to 20 percent of the CoP, attends meetings regularly but not with the regularity or intensity of the core group.

- The peripheral group represents a large portion of the community members. They rarely participate actively but are often not as passive as they seem. They gain their own insights from observing the discussions of the other members of the community.

In a research project the coordinator is the project leader that is in charge of organizing meetings with the people involved in the project. He also writes regularly the meeting report about the project progress.

Last but not least, the practice dimension is related to what each community does. It is specific to each $\mathrm{CoP}$ and is related to the common repository built progressively to support the $\mathrm{CoP}$ functioning. The activity typology of the CoP is described in [5] as: (i) collaboration to solve everyday problems; (ii) dissemination and development of best practices, guidelines and procedures; (iii) building, organisation and management of a body of knowledge; (iv) innovation and creation of breakthrough ideas.

Generally a research project creates new knowledge and is therefore concerned with innovation and creation of breakthrough ideas.

\section{$3 \quad$ Process modelling}

\subsection{Definition of process modelling}

According to [6], a business process can be defined as: "a partially ordered set of enterprise activities that can be executed to achieve some desired end-result in pursuit of a given objective of an enterprise or a part of an enterprise". From this definition we can deduce the definition of a process as a partially ordered set of activities that can be executed to achieve some desired end-result in pursuit of a given objective.

Process modelling describes the task of designing semi-formal, graphical descriptions of processes [7]. Process models are designed using so-called process modelling grammars, i.e., sets of graphical constructs and rules, which define how to combine these constructs [8]. Most available grammars such as UML (Unified Modelling Language) activity diagram or BPMN (Business Process Model Notation) are essentially 
graph-based flowcharting notations that exploit basic shapes such as rectangles or circles and arcs.

The act of building a process model is commonly named the process of process modelling [9, 10]. According to [11], this process consists in three stages: elicitation, modelling and validation.

During elicitation, a natural language (e.g. textual or verbal) description of the problem domain is developed by collecting relevant information objects (e.g., documents, data or informal explanations from process stakeholders), which are then verbalized using a common language. During modelling, these information objects are transformed into a formal specification (i.e. a process model) by mapping the components of the informal specification onto modelling grammar and tool. Last during validation, the model is paraphrased again in natural language in order to be able to validate the resulting text against the natural language description created during the elicitation stage [12].

In other words, process modelling enables to fix the features of a given process enabling to reduce its uncertainty. According to [13] a classical classification of uncertainty is its separation of uncertainty into: aleatory and epistemic. Aleatory uncertainty describes the intrinsic variability associated with a physical system or environment. Epistemic uncertainty, on the other hand, is due to an incomplete knowledge about a physical system or environment. Process modelling deals with Epistemic uncertainty as it enables to pinpoint the lacking knowledge about the process modelled.

\subsection{BPMN}

BPMN (Business Process Model Notation) is a graphical process modelling notation provided by the OMG. According to [14], its objective is to provide businesses with the capability of understanding their internal business procedures in a graphical notation and to give organizations the ability to communicate these procedures in a standard manner. Furthermore, the graphical notation has to facilitate the understanding of the performance collaborations and business transactions between the organizations.

The grammar of BPMN is rich and dedicated to describe in detail business processes. For example, it distinguishes eight different tasks: abstract task, service task, send task, receive task, user task, manual task, business rule task and script task. From this point of view BPMN is a powerful tool to model business processes.

In our view, even if it is dedicated to business processes, it can be applied to any process. We apply it to the modelling of experimental protocols in collaborative research projects. Such a modelling enables the different stakeholders involved to formalize the different steps of the experiments in order to fix it precisely. Such formalization is essential to avoid misunderstanding between the research teams. It also enables to define which experiments each team has to set up and is responsible for. In this way, it avoids the teams the checking of the experimental results of another team. This is essential to save time and money during the project. 


\section{Case study: the SEPOLBE project}

\subsection{Context of the project}

The SEPOLBE project is dedicated to develop bioadmixtures for concrete. These substances are conscientious of the environment and should limit the bio contamination of the concrete surface and improve the resistance to corrosion of its metallic reinforcement. This project implies four research teams and a company. The research teams have different complementary areas of competency: concrete surface analysis, physical chemistry analysis of films on steel and concrete, electro chemistry for steel corrosion inhibition, petro physics for concrete physical chemistry characterization. Competences in micro biology, chemistry and microscopy are also required to develop the substances and to analyse the surface bio contamination. The industrial partner is specialised in concrete admixtures. Its product mix already includes protection products but none of them is dedicated to biological contamination. The duration of this project is about four years. It uses extra cellular substances and has been divided into eight tasks that are scheduled on a GANTT plan providing a shared vision of the project:

- Task 1: project coordination

- Task 2: this task deals with the elaboration and characterization of the products based on the extra cellular products considered. It implies the company and the B2HM team specialized in micro biology. The target is to define the concentration of each extra cellular product in the solution and to optimize its physical chemistry. This task also includes activities linked to the eco profile and security data writing of the different formulas.

- Task 3: this task consists in elaborating test samples of "admixtured" concrete with the products from task two. These samples are then mechanically tested in order to validate the pair (product ingredients, concentration). The admixtures can modify both compressive and bending strength of the mortar samples, but values remain higher than the minimum required by the standard.

- Task 4: this task consists in validating the products' concentration and its influence on the mortar samples properties like setting time. The underlying objective is to check that the adjuvants have no negative impacts on the mortar base properties. This task implies the company and the ICube team having mechanical competencies.

- Task 5: this task consists in validating the inhibiting actions of the selected products. The objective is to choose the products having the best action against bio contamination and corrosion. Here, electro chemistry experiments are worked out by the ICube and LISE teams. The LISE team is specialized in electro chemistry.

- Task 6: this task deals with the understanding of the inhibition corrosion mechanisms exploiting the operative modes defined during the tasks 3, 4 and 5. The LISE and ICube teams are responsible for this task.

- Task 7: this task consists in the characterization of modifications of the concrete surface and the steel/mortar base interfaces. The objective is to determine how the admixtures modify the concrete porous network. Three partners are in charge of 
this task: the ICube team, the company and the LPCS team, specialized in surface physical chemistry.

- Task 8: this task deals with the cleaning ability of the mortar base surfaces. The objective is to evaluate the ability of the bioadmixtures to limit the development of biofilms on the concrete surface and its impact on the cleaning concrete surface (biofilm dropping out). The ICube and B2HM teams are in charge of this task. The ICube team has to provide to the B2HM team "adequate" concrete samples. The $\mathrm{B} 2 \mathrm{HM}$ is in charge of the contamination and cleaning tests.

\subsection{The community of practice}

The SEPOLBE project can be analysed in the light of the CoP theory. This analysis enables the improving of the project functioning towards better collaboration.

The SEPOLBE project is a project based CoP. Indeed, the four research teams and the company work together during 48 months in order to develop bioadmixtures for concrete. After project completion the CoP will be dissolved. This enables the definition of the domain of the CoP.

Concerning the community dimension, for the SEPOLBE project, we can identify a core group and an active group. The core group is represented by the project coordinator and one responsible in each research team involved. The project coordinator which is part of the ICube team checks that the tasks of the project work properly and follow the foreseen schedule. To succeed in project meetings take place twice a year. At least one representative person of each team attends to the meeting.

Concerning the practice dimension the SEPOLBE project mainly share innovative ideas. Indeed, the boadmixtures that have to be developed during the project are new. Therefore, during the project specific communication strategies are set up. Moreover the different stakeholders signed a contract specifying the role of each partner during the project. In this contract the problem of patent rights and scientific publications were stated. Indeed, the industrial partner wants to keep the industrial property on the bioadmixtures that have to be formulated during the project and the scientific partners in the project would like to publish the project results.

\subsection{Process modelling}

We illustrate the usefulness of process modelling on task 8 and the collaboration between the ICube and B2HM teams. The ICube team has to manufacture cement base samples for the B2HM team that proceeds to bio-receptivity tests. The underlying objective is to provide samples whose bio-receptivity remains steady between the manufacturing date and the bio-receptivity tests. The B2HM team requires an average time of two or three weeks to make these tests.

The process parameters that the ICube team checks is the surface roughness, surface $\mathrm{pH}$ value and energy surface and their evolution over time. Indeed, according to these physical chemistry parameters are representative of the bio-receptivity. These preliminary tests aim at validating the manufacturing process and preservation conditions of the samples.

The modelling of the manufacturing process and preliminary test enable the formalization and sharing of the experimental protocols. In this way, the process is codi- 
fied and each partner can rely on this description for its own work. Moreover, the model can be annotated enabling to refine the activity described.

The process model of the tablet manufacturing is given in Fig. 1. First of all the cement base is elaborated according to the standard. This is day zero of the samples. After turning out of the samples, they are stored in distilled water (standard curing conditions). The water/sample relation is steady over time. In this way, the humidity rate is constant (about 100\%) and has therefore not to be taken into account. Then, there is a waiting time from at least two days of cement base hardening (before it is too crumbly to be polished). The polishing enables to have smooth and parallel sides. In this way, the topographical differences linked with casting can be removed. The polish activity uses three different sand papers. Then the samples are ultrasonicated in order to remove the polishing fragments. Both teams discuss on the process model about the preservation conditions and surface condition of the samples. In this way the process epistemic uncertainty is fixed.

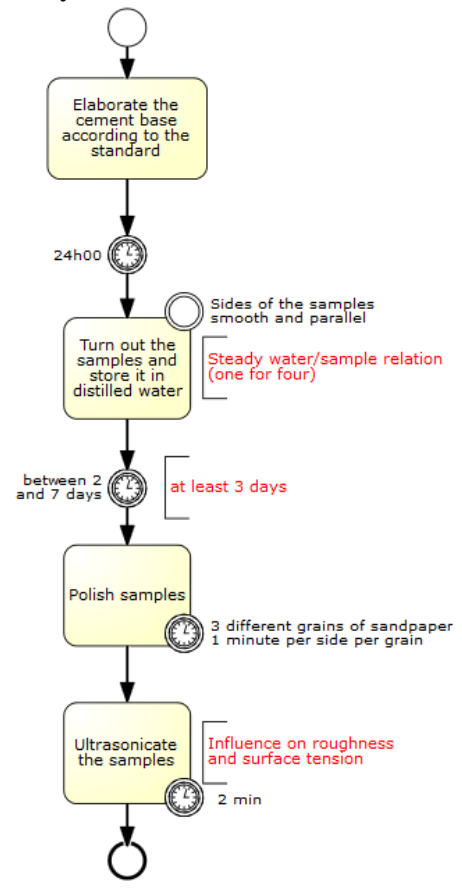

Fig. 1. Cement base sample manufacturing process of the SEPOLBE project

\section{Conclusion}

Research projects are more and more collaborative and multi-disciplinary with teams geographically dispersed. In this boarder, efficient collaborative work has to be ensured. To succeed in we propose to apply two tools: (i) CoP theory and (ii) process modelling. The first one enables the setting up of an appropriate collaboration context by defining the domain, community and practice features. The second one is useful to 
describe experimental protocols on which different teams have to work. BPMN through its rich semantics is particularly adapted, even if it is not dedicated to such a context. The SEPOLBE project shows the applicability of these tools to this particular context. In future it will be interesting to add to the BPMN semantics elements specific to experimental protocol modelling.

\section{$6 \quad$ References}

1. A. Cox, What are communities of practice? A comparative review of four seminal works, Journal of Information Science, vol. 316 pp. 527-540 (2005)

2. E. Wenger, Communities of Practice: Learning, Meaning and Identity, Cambridge University Press, Cambridge, (1998)

3. E. Wenger, R. McDermott, W.M. Snyder, Cultivating communities of practice, Harvard Business School Press, Boston, (2002)

4. R. McDermott, Critical success factors in building communities of practice, Knowledge Management Review, vol. 3 May-June (2000)

5. APQC, Building and Sustaining Communities of Practice: Continuing Success in Knowledge, American Productivity and Quality Centre (APQC), Texas, United States of America, (2001)

6. ISO 19439, Enterprise integration - Framework for enterprise modelling (2006)

7. J.E. Ingvaldsen, J.A. Gulla, Model-based business process mining, Information Systems Management, vol. 23 pp. 19-31 (2006)

8. Y. Wand, R. Weber, Research commentary: information systems and conceptual modeling - a research agenda, Information Systems Research, vol. 13 pp. 363-376 (2002)

9. J. Claes, I.T.P. Vanderfeesten, H.A. Reijers, J. Pinggera, M. Weidlich, S. Zugal, D. Fahland, B. Weber, B. Mendling, G. Poels, Tying process model quality to the modeling process: the impact of structuring, movement, and speed, in the Business Process Management - BPM 2-12.Lecture Notes in Computer Science, Tallinn, Estonia, 2012, pp. 33-48 (Year)

10. M. Indulska, J. Recker, M. Rosemann, P. Green, Process modeling: current issues and future challenge, J.G. P.van Eck, R. Wieringa (Ed.), in the Advanced Information Systems Engineering CAiSE 2009 Lecture Notes in Computer Science, Amsterdam, The Netherlands, 2009, pp. 501-514 (Year)

11. J. Recker, J. Mendling, C. Hahn, How collaborative technology supports cognitive processes in collaborative process modeling: A capabilities-gains-outcome model, Information Systems, vol. 388 pp. 1031-1045 (2013)

12. D.J. Flynn, R. Warhurst, An empirical study of the validation process within requirements determination, Information Systems Journal, vol. 4 pp. 185-212 (1994)

13. J.Y. Dantan, A.J. Qureshi, J.F. Antoine, B. Eisenbart, L. Blessing, Management of product characteristics uncertainty based on formal logic and characteristics properties model, CIRP Ann Manuf Technol, vol. 621 pp. 147-150 (2013)

14. OMG, http://www.bpmn.org/ [last access 27/03/2014], (2014) 